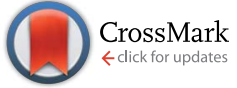

Cite this: RSC Adv., 2015, 5, 46348

Received 9th March 2015

Accepted 18th May 2015

DOI: $10.1039 / c 5 r a 04169 d$

www.rsc.org/advances

\section{Ceramic anion-exchange membranes based on microporous supports infiltrated with hydrated zirconium dioxide}

\author{
M. C. Martí-Calatayud, ${ }^{a}$ M. García-Gabaldón, ${ }^{a}$ V. Pérez-Herranz, ${ }^{\text {a }}$ S. Sales ${ }^{\text {b }}$ \\ and S. Mestre ${ }^{b}$
}

Anion-exchange membranes made from inexpensive ceramic materials were synthesized by using a simple procedure based on incorporating particles of an ion exchanger into a host microporous structure. Microporous ceramics produced from alumina and kaolin were used as supports, and their internal voids were functionalized by direct precipitation of hydrated $\mathrm{ZrO}_{2}$. The addition of starch as pore former to the sintering mixture induces the formation of pores within the micrometer range, where the subsequent deposition of hydrated $\mathrm{ZrO}_{2}$ occurred preferentially. An increase in the loading of hydrated $\mathrm{ZrO}_{2}$ particles improved the ion exchange capacity (IEC) and induced anion-selective properties on the membranes. However, when more than six infiltration cycles were performed, the membrane porosity was substantially reduced. Electrochemical measurements conducted in acidic and neutral media corroborated the implication of the hydrated $\mathrm{ZrO}_{2}$ particles on the development of concentration polarization and confirmed the strong relationship existing between the membrane porosity and electrical conductivity. Conversely, chronopotentiometric curves showed that the membranes practically lack of ion-exchange properties when tested under alkaline conditions. The methodology proposed to synthesize ceramic ion-conducting membranes could significantly broaden the utilization of electromembrane processes in industrial applications where the use of polymeric membranes is restricted due to their expensive cost or poor chemical stability.

\section{Introduction}

The emergence of new electromembrane systems capable of increasing the sustainability of industrial processes has fostered the development of ion-exchange membranes with tailored properties. ${ }^{\mathbf{1 , 2}}$ The role and required characteristics of ion-exchange membranes strongly depend on their final application. ${ }^{3}$ For example, membranes used in polymer electrolyte fuel cells are required to operate at high temperatures, have good ionic conductivities and minimize the fuel crossover. ${ }^{4,5}$ In the field of environmental protection, electromembrane processes are applied to remove ionic contaminants from industrial effluents, thus allowing the reuse of water and the recovery of valuable by-products. ${ }^{6-8}$ However, one of the main limitations of ion-exchange membranes applied to treat industrial effluents is their short durability, which is caused by

${ }^{a}$ IEC Group, Departament d'Enginyeria Quimica i Nuclear, Universitat Politècnica de València, Camí de Vera s/n, 46022, P.O. Box 22012, València, E-46071, Spain. E-mail: vperez@iqn.upv.es; mamarc13@upvnet.upv.es; Fax: +34-96-3867639; Tel: +34-96-3877632

${ }^{b}$ Instituto de Tecnología Cerámica, Campus Universitario Riu Sec, Av. Vicent Sos Baynat s/n, 12006, Castellón, Spain the reduced resistance of polymer materials against chemical degradation by radiation or oxidizing agents. ${ }^{9-11}$

In terms of membrane performance, perfluorosulfonic membranes are considered a reference because they combine excellent ionic conductivities with high chemical stabilities. However, their high cost reduces the viability of using electromembrane processes to treat industrial effluents when compared with other cheaper and less sustainable techniques. ${ }^{\mathbf{1 0}}$ Therefore, the development of chemically resistant ion conducting membranes at reasonable cost remains as one of the major challenges in this field. In the recent time, different approaches have been considered in order to achieve durable membranes. Composite membranes have shown in certain cases interesting properties which combine the advantages of both inorganic and organic materials. ${ }^{12}$ The synthesis of composite materials by dispersing inorganic nanoparticles like $\mathrm{Al}_{2} \mathrm{O}_{3}$ or $\mathrm{SiO}_{2}$ into a polymer matrix has been reported to improve the thermal and mechanical stability of the membranes. ${ }^{13,14}$ An alternative strategy consists on the synthesis of pore-filling electrolyte membranes, which are composed of two types of materials: a porous substrate which restricts the swelling of the polymer and increases the membrane mechanical stability, and a polymer filling the substrate pores and exhibiting ionic conductivity. ${ }^{\mathbf{1 5}}$ 
Ceramic materials exhibit significantly higher thermal and chemical stability in harsh environments than polymers. However, they are generally processed at high temperatures, retain a small number of fixed ionogenic groups and lack of ionexchange properties. ${ }^{16}$ Among the wide variety of ceramic compounds there are important exceptions, such as hydrated oxides of multivalent metals. For example, hydrated oxides of titanium and zirconium polymerize by oxolation and give rise to a dense structure with oxygen atoms at their surface that are not completely coordinated by metal atoms. ${ }^{17}$ These oxygen atoms are associated with water molecules and hydroxyl groups, hence showing ion-exchange properties. In acidic conditions these compounds exchange anions, as indicated as follows:

$$
\mathrm{M}-\mathrm{OH}+\mathrm{X}^{-} \leftrightarrow \mathrm{MX}+\mathrm{OH}^{-}
$$

whereas in alkaline conditions, hydrated oxides can exchange cations:

$$
\mathrm{M}-\mathrm{OH}+\mathrm{A}^{+} \leftrightarrow \mathrm{MOA}+\mathrm{H}^{+}
$$

Therefore, hydrated oxides of multivalent metals are potential compounds for preparing ceramic ion-exchange membranes. Within this group, hydrated zirconium dioxide is a promising candidate because it is stable in acidic and oxidizing media. ${ }^{\mathbf{1 8}}$

The aim of this work is to explore the development of ceramic anion-exchange membranes by applying a facile procedure, which does not require the use of polymeric compounds. Our approach is similar to that used to synthesize pore-filled membranes; however, in the present case both the host supports and the pore-filling particles are ceramic. Microporous substrates synthesized from a mixture of alumina and kaolin were selected as the supports for hosting the ion exchanger. Previous studies have demonstrated the suitability of these supports to act as separators in electrochemical reactors. ${ }^{19,20}$ Moreover, their pore size distribution can be tuned by adding pore formers to the alumina-kaolin mixture..$^{\mathbf{2 0} 21}$ Subsequently, particles of hydrated zirconium dioxide, which provide the membranes with anion-exchange properties, were embedded in the voids of the ceramic supports. The composition, morphology and pore size distribution of the resulting membranes was characterized and their electrochemical behavior was investigated using different electrolyte solutions.

\section{Experimental}

\subsection{Synthesis of the membranes}

The raw materials for the synthesis of the ceramic supports were alumina (AR12B5, Aluminium Pechiney, France), kaolin (ER, Caobar S.A., Spain) and starch (Starch from potatoes, Fluka AG, Germany). The mixture of raw materials (40\%wt alumina, $40 \%$ wt kaolin and $20 \%$ wt starch) was homogenized in a ball mill with distilled water. The resulting suspension was dried under IR lamps. Then, the powder was moisturized to a final water content of $4 \mathrm{~kg} \mathrm{H} \mathrm{H}_{2} \mathrm{O} / 100 \mathrm{~kg}$ dry solid using a solution consisting of $4 \%$ wt of poly-vinylalcohol (PVA) in distilled water. PVA acted as a binder between the ceramic particles.

Cylindrical test specimens were obtained by unidirectional dry pressing at $400 \mathrm{~kg} \mathrm{~cm} \mathrm{~cm}^{-2}(50 \mathrm{~mm}$ diameter and $3 \mathrm{~mm}$ thickness). The green supports were dried in an oven at $110{ }^{\circ} \mathrm{C}$ and then submitted to a thermal treatment designed to completely oxidize the starch particles in an electric furnace (Mod K60L, Nannetti, Italy). Finally, the supports were sintered in an electric kiln (RHF1600, Carbolite Furnaces Ltd.) using a thermal cycle which consisted of a ramp at $25{ }^{\circ} \mathrm{C} \mathrm{min}{ }^{-1}$ until reaching $1400{ }^{\circ} \mathrm{C}$, and a period of 6 hours during which this temperature was kept constant. The supports showed no mass loss in a test of acid resistance and a mass loss of $1.78 \%$ as a result of a test of alkali resistance (12 days in contact with $\mathrm{HCl}$ $18 \%(\mathrm{v} / \mathrm{v})$ or $\mathrm{KOH}$ solution of $100 \mathrm{~g} \mathrm{~L}^{-1}$ respectively, according to standard ISO 10545-13: 1998). In consequence, no changes in the microstructure were expected as a result of the contact of the membranes with the solutions employed in the experiments.

Hydrated zirconium dioxide was deposited in the porous network of the membranes by direct precipitation. First, the ceramic supports were partially immersed for 30 minutes in a $0.5 \mathrm{M} \mathrm{ZrOCl}_{2} \cdot 8 \mathrm{H}_{2} \mathrm{O}$ aqueous solution (98\%, Sigma Aldrich $\mathrm{GmbH}$, Germany), so that only the lower surface of the supports was in contact with the liquid and the solution could be sucked by the porous support. Then, the solution-saturated supports were immersed in a $\mathrm{NH}_{4} \mathrm{OH}$ solution (28-30\%wt, Sigma Aldrich $\mathrm{GmbH}$, Germany) for 24 hours. Subsequently, the membranes were thoroughly washed with distilled water, dried in an electrical oven at $110{ }^{\circ} \mathrm{C}$ until a constant weight was reached. Finally, the membranes were thermally treated at $200{ }^{\circ} \mathrm{C}$ for 30 minutes in order to fix the hydrated zirconium dioxide to the surface of the pores. The infiltration process was repeated up to eight times, and the number of infiltration cycles was denoted as $n$.

The mass of the membranes after each infiltration cycle was recorded and the progress of the infiltration process was evaluated by calculating the parameter $\lambda$, defined as shown in eqn (3):

$$
\lambda=\frac{\text { mass of hydrated } \mathrm{ZrO}_{2} \text { deposited }}{\text { initial mass of the support }}
$$

\subsection{Chemical and morphological characterization of the membranes}

The mineralogical composition of the membranes was obtained by X-ray diffraction (XRD, D8 Advance, Bruker AXS Inc, USA). The morphology and composition of the membranes was analyzed in polished sections by Field Emission Gun-Scanning Electron Microscopy (FEG-SEM, QUANTA 200F, FEI Co, USA). The distribution of zirconium inside the membranes was analyzed by Energy-dispersive X-ray spectroscopy (EDX, Genesis 7000 SUTW, EDAX, USA) connected to the SEM. The composition of different sections $(2500 \mu \mathrm{m} \times 250 \mu \mathrm{m})$ was analyzed with EDX at different distances from the surface of the membrane to obtain the profile of $\mathrm{Zr}$ weight percentage across the membranes. For each distance, two screens were taken and the 
average of the two measurements was obtained. Mercury intrusion porosimetry (AutoPore IV 9500 porosimeter, Micromeritics Instrument Co, USA) was used to measure the pore size distribution.

\subsection{Electrochemical characterization of the membranes}

2.3.1. Ion exchange capacity. The IEC indicates the amount of fixed charges present in the membrane structure per weight of total membrane material, i.e.: the total active sites capable of exchanging solution counterions (electrolyte ions of opposite charge sign). This parameter is useful in order to compare ionexchange membranes obtained from different manufacturing conditions. The IEC is calculated by means of successive equilibrating steps in which the ions balancing the membrane fixed charges are replaced by other type of ions present in an equilibrating solution. The membranes were first immersed during $24 \mathrm{~h}$ in $0.05 \mathrm{M} \mathrm{NaOH}$. Subsequently, they were rinsed in abundant distilled water in order to remove the $\mathrm{NaOH}$ not bound to the fixed charges, and were immersed during $24 \mathrm{~h}$ in $0.01 \mathrm{M} \mathrm{HCl}$. The excess of $\mathrm{Cl}^{-}$ions in solution replace the $\mathrm{OH}^{-}$ ions that are initially balancing the membrane fixed charges. Therefore, the amount of membrane fixed charges can be obtained by calculating the change in acidity in the $\mathrm{HCl}$ solution occurred due to the release of $\mathrm{OH}^{-}$ions by the membrane. This quantity is then divided by the dry weight of the membranes in order to obtain the ion exchange capacity.

2.3.2. Chronopotentiometry. Chronopotentiometry is an electrochemical technique used to characterize the dynamics of the ion transport occurring through a system formed by an ionexchange membrane and the surrounding electrolyte. It is based on the application of current pulses and the registration of the evolution of the voltage drop of the membrane system $\left(U_{\mathrm{m}}\right)$. An electrochemical cell composed of 3 compartments, which is described in a previous study, ${ }^{22}$ was used for this purpose. Each compartment has a volume capacity of $130 \mathrm{ml}$ and upper apertures for the installation of the electrodes. The synthesized ceramic anion-exchange membranes (with an effective area of $3.52 \mathrm{~cm}^{2}$ ) were clamped between the anodic and the central compartment, whereas an auxiliary cation-exchange membrane (Nafion 117, DuPont) was installed between the central and the cathodic compartment. Two graphite bars were used as cathode and anode and were connected to a potentiostat/galvanostat (Autolab®, PGSTAT 20), used as power supply. Two Ag/AgCl reference electrodes were used to measure the voltage drop of the membrane system, $U_{\mathrm{m}}$, which is composed of the membrane itself and the adjacent solution layers. The reference electrodes were immersed into two Luggin capillaries installed at each side of the membrane with their tips facing each other and placed at approximately $1 \mathrm{~mm}$ from the membrane surface. The experiments were conducted at room temperature $\left(25^{\circ} \mathrm{C}\right)$ and without stirring. The same solution was placed in the three compartments in order to minimize the influence associated with concentration differences between compartments. ${ }^{23}$ Prior to conducting the experiments the membranes were equilibrated during $24 \mathrm{~h}$ in the same solutions to be investigated subsequently. The current pulses were applied during $300 \mathrm{~s}$. In addition to the chronopotentiometric measurements, the current-voltage curves of the membranes were obtained by plotting the values of $U_{\mathrm{m}}$ registered at the end of each current pulse against the corresponding values of current density. These curves provide useful information about the steady state behavior of the membranes over different ranges of current density.

The electrochemical behavior of the membranes at an acidic $\mathrm{pH}(\mathrm{pH} \sim 1.3)$ was studied using $0.05 \mathrm{M} \mathrm{HCl}$ solutions (J.T. Baker®). NaCl solutions (Panreac $\left.{ }^{\circledR}\right)$ were used to evaluate the membranes in neutral media. In that case, the effect of the electrolyte composition was also studied by using different concentrations of $\mathrm{NaCl}(0.01 \mathrm{M}, 0.05 \mathrm{M}$ and $0.1 \mathrm{M})$. In order to study the membrane behavior with the same electrolyte counterions $\left(\mathrm{Cl}^{-}\right)$but under alkaline conditions, additional experiments were conducted using mixtures of $0.05 \mathrm{M} \mathrm{NaCl}$ and $0.05 \mathrm{M} \mathrm{NaOH}(\mathrm{pH} \sim 12.7)$.

\section{Results and discussion}

\subsection{Chemical and morphological characterization}

The sintered supports were mainly formed by corundum $\left(\alpha-\mathrm{Al}_{2} \mathrm{O}_{3}\right)$ and mullite $\left(\mathrm{Al}_{6} \mathrm{Si}_{2} \mathrm{O}_{13}\right)$, with a minor proportion of cristobalite in some samples ( $\mathrm{SiO}_{2}$ allotrope). These phases ensure high chemical resistance to the support (Fig. 1). The membrane also showed an amorphous halo in the range of $2 \theta$ $\left[27^{\circ}-35^{\circ}\right]$ that was identified as non-crystalline hydrated zirconium dioxide by comparison with a previously prepared sample of the ion exchanger. This sample was obtained as a precipitate from the same reagents and heat treated with the same thermal cycle employed to fix the exchanger to the support. The sample showed the same amorphous halo, but in case of treating it to $550{ }^{\circ} \mathrm{C}$ results in anhydrous zirconium dioxide. This result agrees with the crystallization temperature of hydrated $\mathrm{ZrO}_{2}$ described in the literature (around $450{ }^{\circ} \mathrm{C}$ ), ${ }^{24}$ and with the fact that $200{ }^{\circ} \mathrm{C}$ was the maximum temperature of the thermal treatment employed to fix the ion-exchanger to the support.

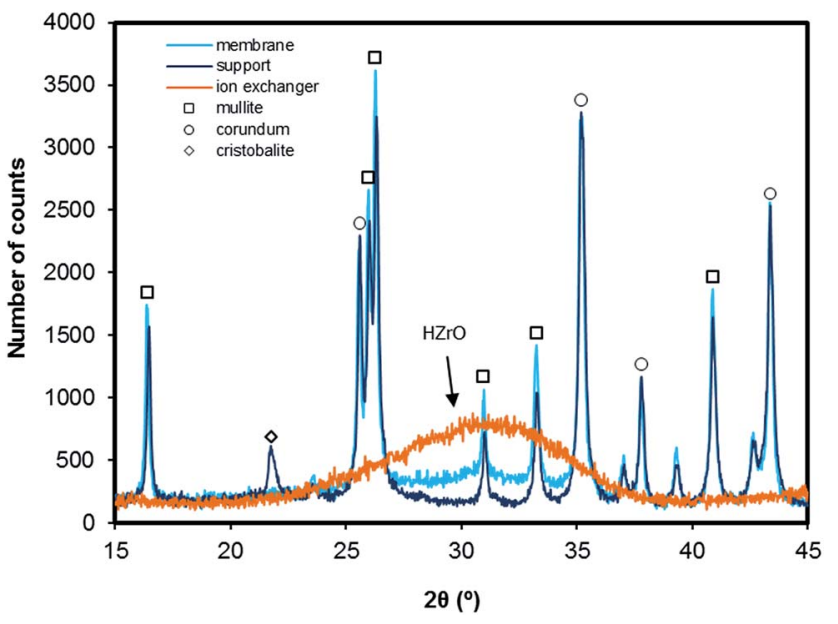

Fig. 1 Diffractograms of the ceramic supports, the ion exchanger, and the membranes obtained after several infiltration cycles with hydrated $\mathrm{ZrO}_{2}$. 
FEG-SEM images of the unmodified microporous supports and the membrane obtained after 8 infiltration cycles are shown in Fig. 2. As can be seen from comparing Fig. 2(a) and (c), the hydrous zirconium dioxide was distributed throughout the entire porous network of the infiltrated membrane. However, the amount of hydrous zirconium dioxide seemed to be higher in the larger pores (white dots in Fig. 2(c)). The observation of the membranes at higher magnification showed that hydrated zirconium dioxide was deposited as sub-micron particles forming a dense layer at the inner surface of the pores (Fig. 2(d)).

Fig. 3 shows the profile of $\mathrm{Zr}$ weight percentage across the section of the membrane submitted to eight infiltration cycles (evaluated as $\mathrm{ZrO}_{2}$ ). Although a small increase of the zirconium content was detected near the surface which had been in contact with the $\mathrm{ZrOCl}_{2} \cdot 8 \mathrm{H}_{2} \mathrm{O}$ solution, an appreciable amount of the ion exchanger was present throughout the membrane section. Accordingly, it can be considered that the infiltration process achieved an acceptable distribution of the hydrated zirconium dioxide.

The pore size distribution of the different synthesized membranes is represented in Fig. 4. The unmodified microporous supports have a trimodal pore size distribution, i.e. with three different characteristic pore diameters $(\sim 0.09$, $\sim 0.44$ and $\sim 1.75 \mu \mathrm{m})$. The effect of the starch addition on similar alumina-kaolin ceramic supports was investigated in a previous study, and it was observed that the interconnection of small pores occurring when the starch particles are burnt provoked the formation of pores of higher diameter $(\sim 1.75 \mu \mathrm{m}){ }^{19}{ }^{1}$ For the membranes modified with up to 4 infiltration cycles ( $n=2$ and $n=4$ in Fig. 4$)$, the characteristic

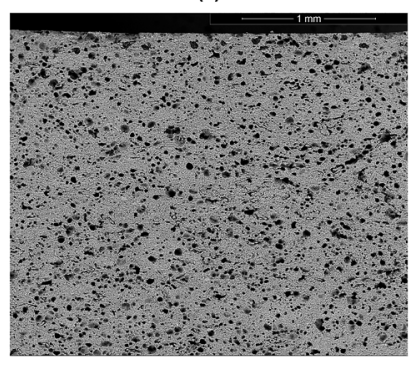

(c)

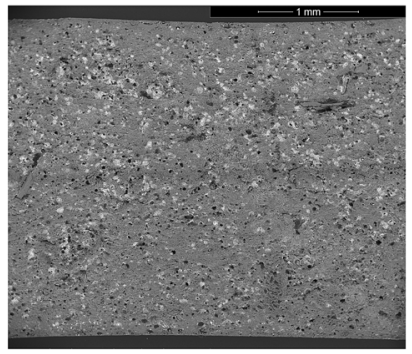

(b)

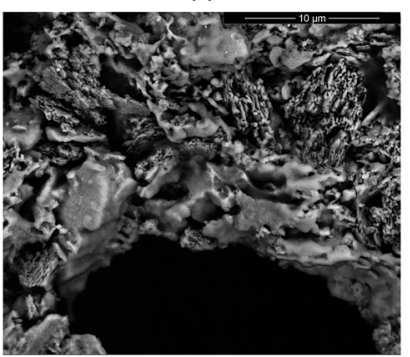

(d)

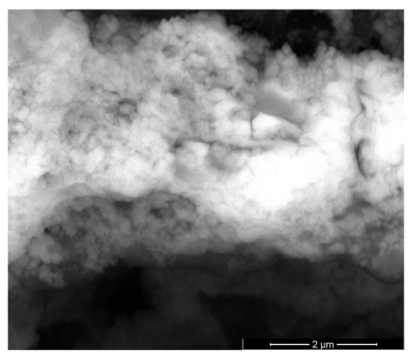

Fig. 2 FEG-SEM images of polished sections of the membranes (a) cross section of the unmodified microporous support, (b) amplification of the inner pores surface of the unmodified microporous support, (c) cross section of the membrane with $n=8$, and (d) amplification of the inner pores surface of the membrane with $n=8$.

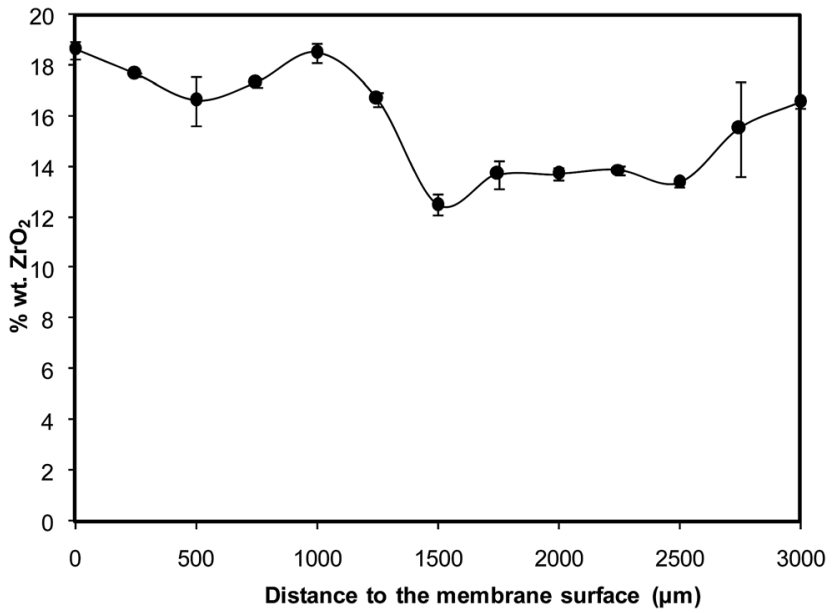

Fig. 3 Concentration profile of $\mathrm{ZrO}_{2}$ along the cross section of the membrane obtained after eight infiltration cycles.

diameter of the highest pores diminishes gradually (from $\sim 1.75 \mu \mathrm{m}$ for $n=0$ to around $0.7 \mu \mathrm{m}$ for $n=4$ ). On the contrary, the two smaller characteristic pore diameters remain approximately unchanged. These results suggest that for the first infiltration cycles, the particles of hydrated $\mathrm{ZrO}_{2}$ are preferentially deposited at the walls of the largest membrane pores. The membranes resulting from a higher number of infiltration cycles ( $n=6$ and $n=8)$ only show two characteristic pore diameters. The change from a trimodal to a bimodal pore size distribution may be due to the further inclusion of ion exchanger inside the larger pores, whose diameter becomes smaller and overlaps with the peak corresponding to the intermediate characteristic pore diameter $(\sim 0.44 \mu \mathrm{m})$. Finally, for the membranes with $n=8$ the largest characteristic pore size decreases from $\sim 0.44$ to $0.35 \mu \mathrm{m}$, thus confirming that the amount of ion exchanger deposited inside the porous membranes increases gradually with the number of infiltration cycles and contributes to decrease the porosity of the membranes.

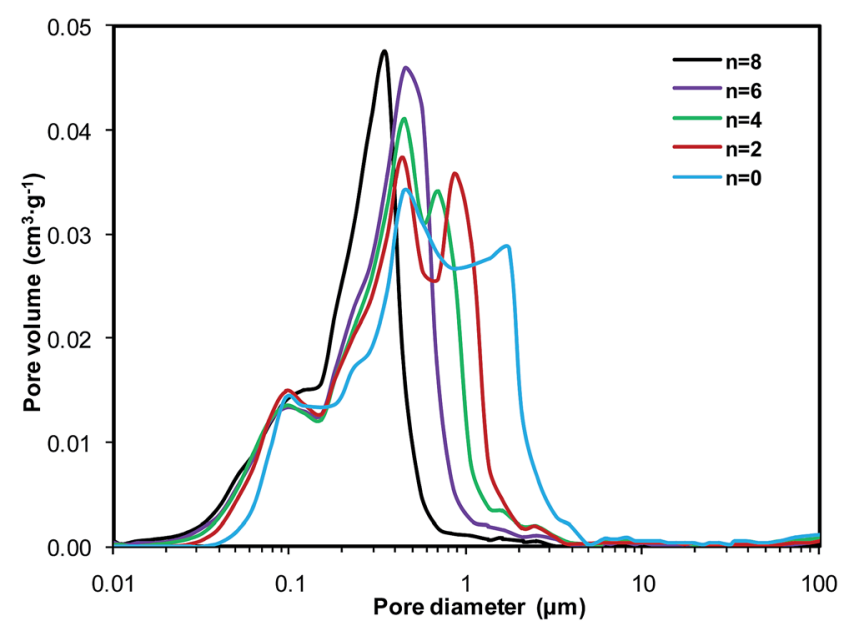

Fig. 4 Effect of the number of infiltration cycles with hydrated $\mathrm{ZrO}_{2}$ on the pore size distribution of the membranes. 
The obtained results indicate that the particles of hydrated $\mathrm{ZrO}_{2}$ are infiltrated predominantly in the largest pores. Conversely, the pattern of infiltration exhibited by other types of ion exchanger can differ significantly from that obtained for hydrated $\mathrm{ZrO}_{2}$. Specifically, the deposition of zirconium phosphate was reported to occur uniformly in pores of different diameters. ${ }^{19}$ Therefore, it seems that in the present case the addition of starch during the formation of the microporous supports is essential in order to create pores of an optimum diameter where the deposition of ion exchanger takes place preferentially. Moreover, from the evolution of the pore size distributions with $n$, it can be concluded that the $\mathrm{ZrO}_{2}$ is significantly deposited over previously infiltrated $\mathrm{ZrO}_{2}$ particles. Other researchers have also observed that the deposition of hydrated $\mathrm{ZrO}_{2}$ as pore filler can promote the transformation of ceramic macropores into a secondary nanoporous ionconductive structure, which results from the aggregation of several $\mathrm{ZrO}_{2}$ nanoparticles. ${ }^{25}$

\subsection{Electrochemical behavior of the membranes}

3.2.1. Ion exchange properties. The application of a higher number of infiltration cycles implied a gradual increase of the amount of ion exchanger deposited in the structure of the ceramic supports, as denoted by the linear increase of $\lambda$ shown in Fig. 5(a) and also by the continuous decrease of the characteristic pore sizes. These results indicate that the amount of deposited ion exchanger can be gradually increased by repeating the infiltration process and forming larger aggregates of ion exchanger. However, the constant increase in the amount of hydrated $\mathrm{ZrO}_{2}$ embedded in the membrane does not necessarily entail an improvement of the membrane ion exchange properties, since a maximum in IEC is reached for $n=6$ (0.07 meq $\mathrm{g}^{-1}$ ) and, instead of following the same linear increase observed for $\lambda$, this parameter is not improved for $n=8$ (see Fig. 5(b)). The evolution of IEC with the number of infiltration cycles indicates that there is a limit from which the increase in the amount of ion exchanger implies a reduction in the active surface available for the exchange of counterions. Accordingly, in terms of IEC, $n=6$ seems to be the optimum number of infiltration cycles to obtain anion-exchange membranes. X. Li et al. also reported a maximum in IEC when the weight percentage of $\mathrm{ZrO}_{2}$ of composite anionexchange membranes was increased. ${ }^{26}$

The obtained IEC values are very low in comparison with typical values reported for commercial polymeric membranes $\left(\sim 1-2\right.$ meq $\left.\mathrm{g}^{-1}\right)$. This significant difference between membranes produced from different materials can be explained by the lower density of polymers in comparison with ceramics. However, the range of IEC values is slightly higher than that of other ceramic ion conducting membranes presented in previous studies. ${ }^{25,27,28}$ Moreover, the anion-exchange capacity of the membranes is in agreement with the values of $\lambda$, if the anion-exchange capacity of hydrated $\mathrm{ZrO}_{2}$ particles reported in previous studies is considered ( $\leq 1 \mathrm{meq}^{-1}$ at acidic $\left.\mathrm{pH}\right) .{ }^{17}$

According to the results above presented the process of modification of ceramic supports is schematically depicted in Fig. 6 . The pores generated by the particles of starch during the sintering process allow the interconnection of different pores. These pores will subsequently serve as host structures for embedding the particles of ion exchanger. In addition to this, the successive repetition of additional infiltration cycles seems to lead to a complete coverage of the pore walls and induces the formation of agglomerates of hydrated $\mathrm{ZrO}_{2}$. Consequently, the (a)

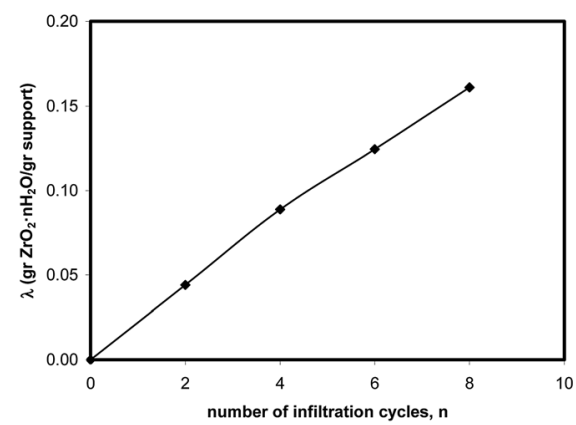

(b)

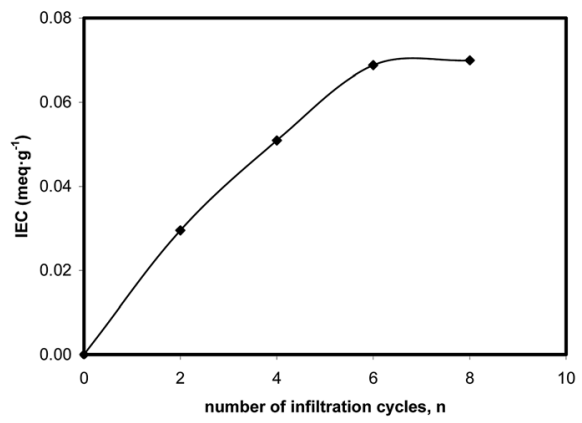

Fig. 5 Effect of the number of infiltration cycles with hydrated $\mathrm{ZrO}_{2}$ on (a) $\lambda$ and (b) IEC.

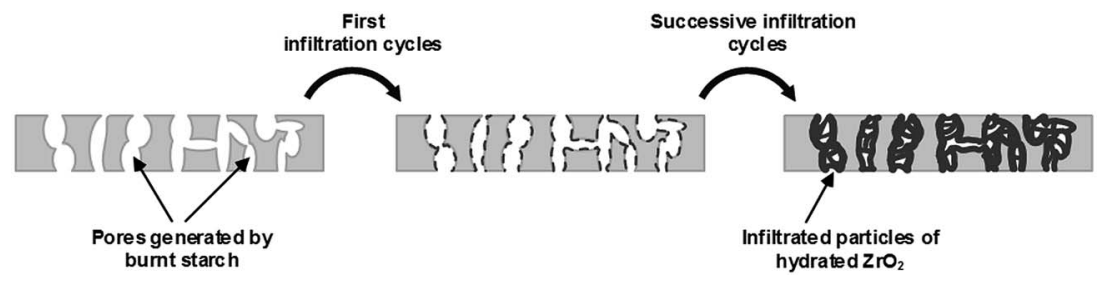

Fig. 6 Schematic representation of the modification of the microporous ceramic supports with hydrated $\mathrm{ZrO}_{2}$. 
amount of ion exchanger and the porosity of the resulting membranes can be modulated by optimizing the number of infiltration cycles.

3.2.2. Chronopotentiometric response. The chronopotentiometric experiments are useful to get a qualitative understanding of the ion transport occurring across the membranes due to the application of an external electric field. Fig. 7 shows the chronopotentiograms obtained with the uncharged ceramic supports and the infiltrated anion-exchange membranes using $0.01 \mathrm{M} \mathrm{NaCl}$ as the electrolyte. At the lowest values of current density, the response obtained is practically flat for all the membranes tested: at the instant where the current is switched on, $U_{\mathrm{m}}$ increases sharply from zero and attains a value which remains practically constant during the course of the current pulse. This response is typical of a quasiohmic membrane behavior, which is characterized by the absence of important mass transport limitations in the membrane system.

However, as the current is increased, there is a clear difference between the curves obtained for the ceramic support and those obtained for the charged membranes. Whereas the response of the unmodified support is almost flat with independence of the applied current, the curves of the infiltrated membranes show a notorious variation of $U_{\mathrm{m}}$ during the application of the current pulses. At a specific time $\tau$, known as the transition time (see Fig. 7(b)), the curves exhibit an inflexion point and $U_{\mathrm{m}}$ increases notably until its value levels off again. This behavior is typical of membranes with ion-selective properties, and is associated with the variations in the electrolyte concentration occurring at the membrane/solution interfaces when high driving forces (current densities) are applied. The facilitated transport of counterions through the membrane leads to the formation of gradients of concentration. This phenomenon is conventionally called concentration polarization in the field of membrane science. ${ }^{29}$ When the concentration of counterions (in this case $\mathrm{Cl}^{-}$ions) drops to very low values at the depleting membrane surface, the electrical resistance of the membrane system increases, and this is reflected in the chronopotentiograms by the sharp increase of $U_{\mathrm{m}}$ with time.

For a constant concentration of $\mathrm{NaCl}$ there is an important effect of the number of $\mathrm{ZrO}_{2}$ infiltration cycles on the chronopotentiograms. As the amount of ion exchanger embedded in the membranes increases, the increase in $U_{\mathrm{m}}$ associated with the concentration polarization becomes more notorious (e.g.: compare the curves obtained at $0.57 \mathrm{~mA} \mathrm{~cm}^{-2}$ in Fig. 7(c) and (d)). In addition, for the highest values of current, the contribution to $U_{\mathrm{m}}$ due to the concentration polarization becomes comparably higher than the quasi-ohmic contribution registered at the beginning of the pulses. These results indicate that the counterions are more easily transported through the (a)

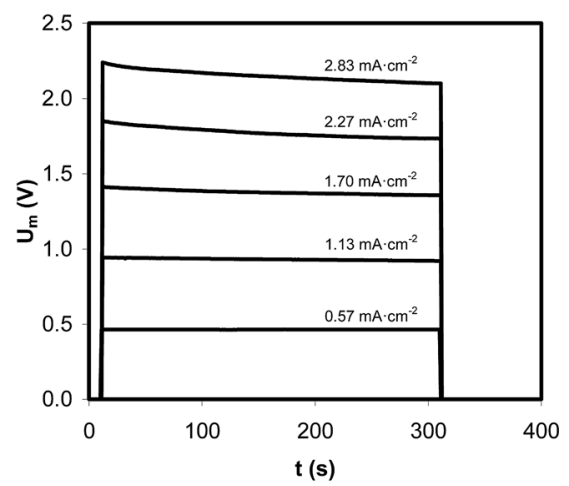

(c)

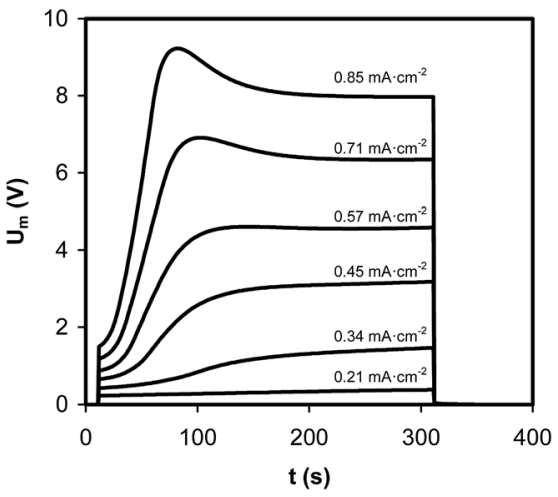

(b)

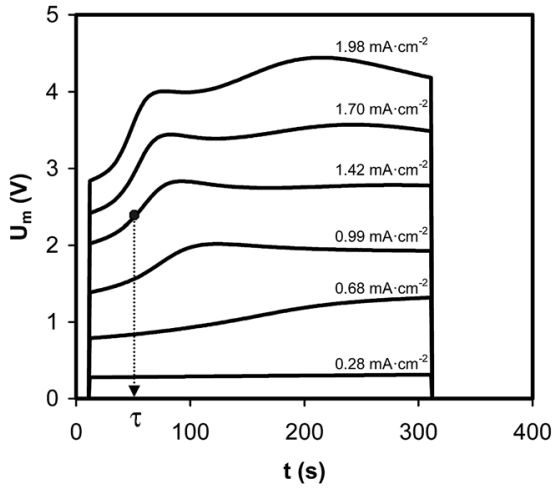

(d)

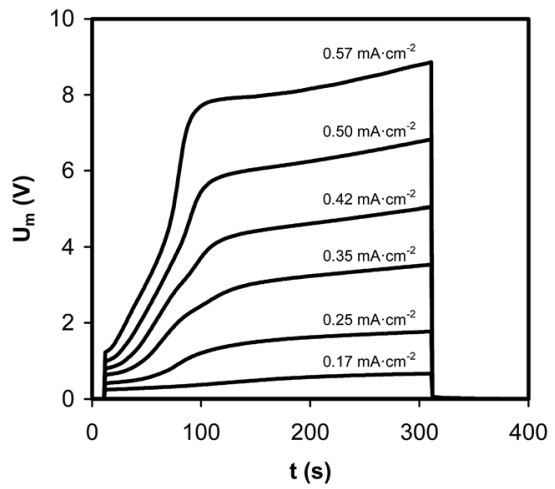

Fig. 7 Chronopotentiometric curves obtained for $0.01 \mathrm{M} \mathrm{NaCl}$ with (a) the microporous support without $\mathrm{ZrO}_{2}(n=0)$, and the membranes infiltrated with hydrated $\mathrm{ZrO}_{2}$ of (b) $n=2$, (c) $n=4$, and (d) $n=6$. 
membranes having a higher content of hydrated $\mathrm{ZrO}_{2}$, which causes a more notorious concentration drop at the depleting membrane surface. However, this phenomenon also implies higher voltage drops (i.e. higher electrical resistances) for the charged membranes when high current densities are applied.

In order to explain the different results obtained for the unmodified supports and the infiltrated membranes, a schematic representation of the mass transport occurring through their structure has been depicted in Fig. 8. Since the microporous supports lack of fixed charges, the current transfer through their structure is associated with the transport of both anions and cations, similarly as occurs in the electrolyte solution (Fig. 8(a)). However, as the number of fixed charges attached to the membranes increases, the ion transport through the membranes becomes predominantly unidirectional, i.e. the anions are attracted by the membrane fixed charges and cross the membrane toward the anodic compartment driven by the electric field whereas the cations present in the anodic compartment are repelled by the positively charged $\mathrm{ZrO}_{2}$ particles (Fig. 8(b)). The enhanced selectivity of the membranes caused by an increasing number of fixed charges may be the reason for the differences observed in the chronopotentiograms. In the case of the unmodified microporous supports the transport rate of species at both the solution and the membrane phase are similar and the variation in the concentration of species in the vicinities of the membranes are rather small, thus leading to flat chronopotentiometric responses. On the contrary, the transport of counterions (i.e. $\mathrm{Cl}^{-}$) occurs faster through the infiltrated membranes than in the solution, whereas the transport of co-ions (i.e. $\mathrm{Na}^{+}$) through the membranes is hindered. This leads to the continuous depletion of ions at the cathodic side of the membranes and produces an accumulation of ions at the anodic side. If the electric field is intensified, the concentration gradients become stronger and a limiting condition can be achieved when the concentration practically drops to zero near the depleting membrane surface. This phenomenon occurs when the limiting current density $\left(i_{\text {lim }}\right)$ has been reached and corresponds to the chronopotentiograms where the increase in $U_{\mathrm{m}}$ is registered. The correlation between the applied current density, the effects on the concentration gradients generated near the anion-exchange membranes and the chronopotentiometric response is schematically shown in Fig. 8(c).

The effect of the electrolyte concentration and $\mathrm{pH}$ on the electrochemical behavior of the membranes was also investigated. Fig. 9 shows the different responses obtained for the membrane with $n=6$ in more concentrated $\mathrm{NaCl}$ solutions and in acidic and alkaline solutions. The shape of the chronopotentiograms obtained for higher $\mathrm{NaCl}$ concentrations (Fig. 9(a) and (b)) is analogous to that presented previously for $0.01 \mathrm{M} \mathrm{NaCl}$ (Fig. 7(d)). The membrane shows the behavior typical of commercial ion-exchange membranes for the three concentrations tested. However, it has to be noted that for similar membrane voltage drops, the current transferred through the membranes increases with the electrolyte concentration. These results reveal the important effect that the electrolyte composition has on the electrical conductivity of the different membrane systems. The curves obtained with $\mathrm{HCl}$ solutions also present an analogous response (Fig. 9(c)), hence confirming that the membranes possess anion-exchange properties at acidic $\mathrm{pH}$ values, as predicted by eqn (1). (a)

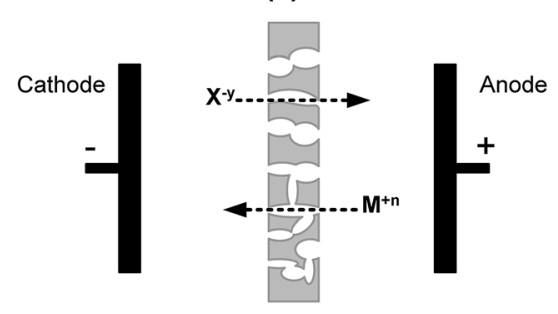

(c) (b)

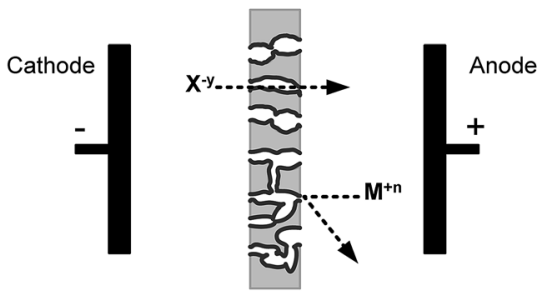

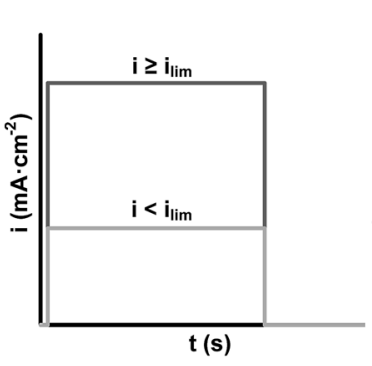

Application of current pulses

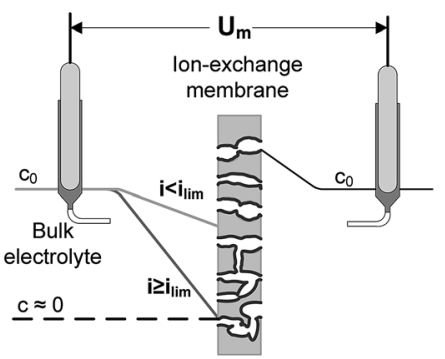

Concentration polarization

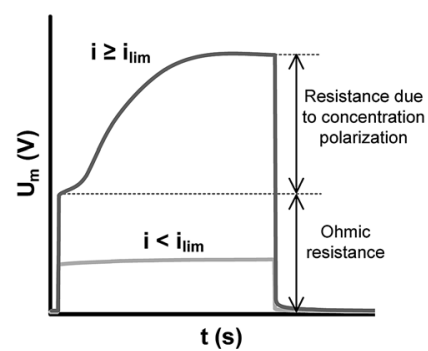

Chronopotentiograms

Fig. 8 Scheme of the ion transport taking place through the different ceramic membranes: (a) transport of positive and negative ions through the uncharged porous supports, (b) transport of counterions (anions) through the charged membranes, and (c) relationship existing between the input signal, the development of concentration polarization in a membrane/electrolyte system and the resulting response registered during the conduction of chronopotentiometric experiments. 
(a)

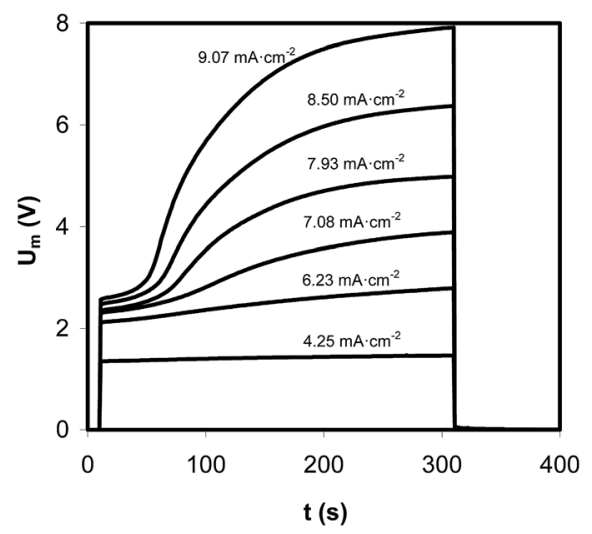

(c)

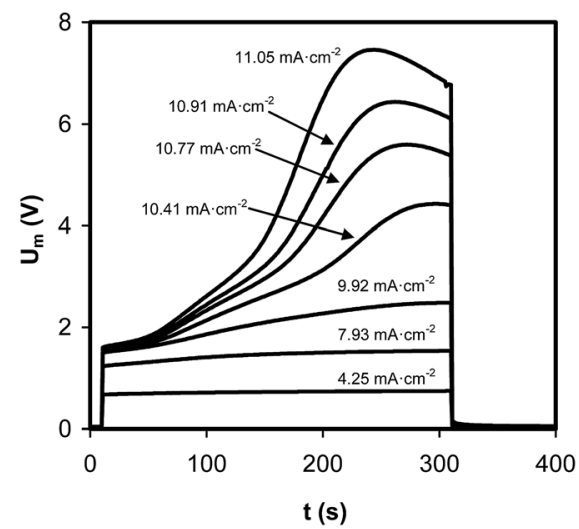

(b)

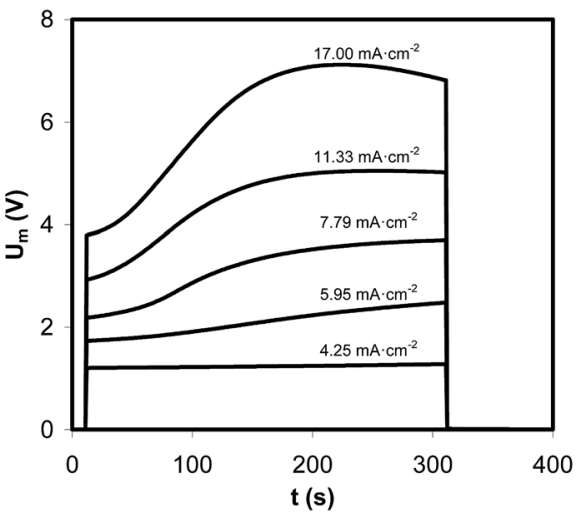

(d)

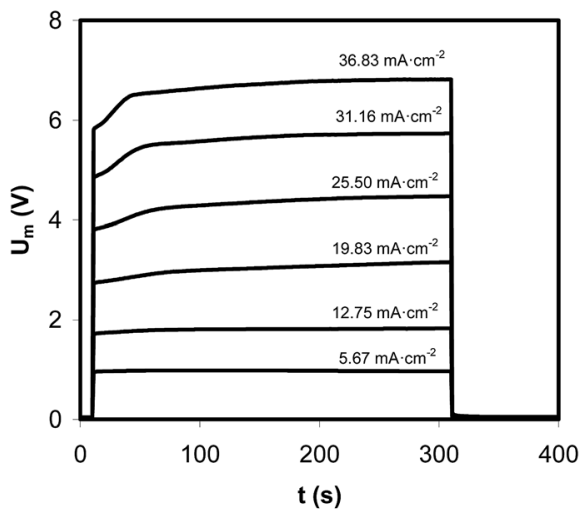

Fig. 9 Chronopotentiometric curves obtained for the membrane obtained after 6 infiltration cycles with different electrolyte solutions: (a) 0.05 $\mathrm{M} \mathrm{NaCl}$, (b) $0.1 \mathrm{M} \mathrm{NaCl}$, (c) $0.05 \mathrm{M} \mathrm{HCl}$, and (d) mixtures of $0.05 \mathrm{M} \mathrm{NaCl}$ and $0.05 \mathrm{M} \mathrm{NaOH}$.

When the membranes are tested with a basic electrolyte $(0.05$ $\mathrm{M} \mathrm{NaCl}+0.05 \mathrm{M} \mathrm{NaOH}, \mathrm{pH} \sim 12.7$ ), the main contribution to the membrane voltage drop is of the quasi-ohmic type while the increase in $U_{\mathrm{m}}$ associated with the concentration polarization is practically negligible. In this case the curves are rather similar to those obtained for the uncharged microporous supports, and therefore, these results suggest that under basic conditions the membranes lose their selectivity. The loss of selectivity of the membranes under alkaline conditions is in agreement with previous studies conducted with $\mathrm{ZrO}_{2}$ particles, which indicated that they exhibit an amphoteric behavior and lose their capability of exchanging anions at basic $\mathrm{pH} .{ }^{27}$ In addition, previous research work has also revealed that the capacity of the hydrated $\mathrm{ZrO}_{2}$ for exchanging cations under alkaline conditions is significantly lower than its capacity for exchanging anions in acidic media. ${ }^{17}$

3.2.3. Current-voltage curves. In addition to the evaluation of the dynamics of ion transport occurring through the membranes at different current densities, chronopotentiometry is also useful to obtain the current-voltage relationship for the different membrane/electrolyte systems. Fig. 10 sows the current-voltage curves obtained for $0.01 \mathrm{M} \mathrm{NaCl}$ solutions. In this graphic it can be seen that the current-voltage curve obtained for the uncharged microporous support exhibits a linear trend within the entire range of current densities tested, where their electrical conductivity remains nearly constant. As mentioned previously, the results obtained with the supports are indicative of the absence of important mass transfer limitations at the membrane/electrolyte interfaces. This trend is in agreement with the flat chronopotentiograms obtained for the supports, hence confirming their lack of selectivity for either cations or anions.

On the contrary, the curves obtained for the infiltrated membranes show a different behavior depending on the range of current density. At low values of current density, the relationship between the current and the membrane voltage drop also corresponds to a quasi-ohmic behavior. However, when the current densities reach values close to the corresponding $i_{\text {lim }}$ the resistance of the membrane system increases and the curves bend forming a plateau. This feature is related to the intensification of concentration polarization, which is consequence of the membrane selectivity. In addition, the curve obtained for the membrane corresponding to $n=2$ exhibits a third zone at currents higher than the $i_{\text {lim }}$ where the current increases again with $U_{\mathrm{m}}$. This third zone, which is usually called the overlimiting region, was not obtained for the membranes with a higher content of $\mathrm{ZrO}_{2}$. 


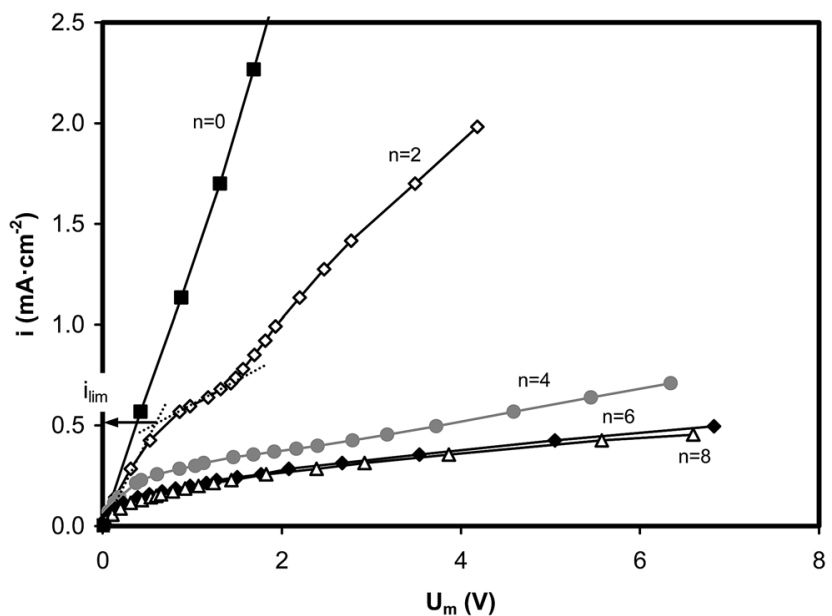

Fig. 10 Current-voltage curves obtained for $0.01 \mathrm{M} \mathrm{NaCl}$ solutions.

The registration of overlimiting current densities is usually attributed to the activation of the supply of ions from the bulk solution to the membrane surface via the onset of convective phenomena by an intense electric field (i.e. onset of electroconvection and gravitational convection). ${ }^{30}$ However, in the present case, the different behavior shown by the membrane corresponding to $n=2$ and the membranes with $n=4,6$ and 8 indicates that there is an important effect of the IEC values on the emergence of this third region in the curves. In the present case, the amount of hydrated $\mathrm{ZrO}_{2}$ embedded in the membrane with $n=2$ seems to be insufficient to maintain the selectivity for anions at high current densities. Therefore, when a strong electric field is applied, co-ions ( $\mathrm{Na}^{+}$ions) may overcome the repulsive forces exerted by the fixed charges, thus promoting the bidirectional transport of anions and cations through the membranes. Conversely, membranes with higher IEC values are capable of maintaining the selectivity even for values of current density higher than the $i_{\text {lim }}$, as confirmed by the long plateau regions registered for these membranes. Membranes having higher loadings of ion exchanger, instead of allowing an important transport of co-ions through their structure at high current densities, maintain the preferential transport of counterions and facilitate the continuous depletion of the electrolyte layer present at the cathodic side of the membrane. The curves obtained for higher concentrations of $\mathrm{NaCl}$ are similar to those presented in Fig. 10, and have been omitted for the sake of conciseness.

Fig. 11 shows the current-voltage curves obtained using $0.05 \mathrm{M} \mathrm{HCl}$ solutions. The shape of the curves is analogous to that obtained with $\mathrm{NaCl}$. The response corresponding to the unmodified ceramic supports is of the quasi-ohmic type within the entire range of currents tested. However, in this case all the membranes infiltrated with hydrated $\mathrm{ZrO}_{2}$ present two characteristic regions: the quasi-ohmic region and the plateau associated with the concentration polarization phenomena. Unlike the results obtained with $\mathrm{NaCl}$, in this case the membrane with $n=2$ does not exhibit an overlimiting region. This discrepancy showed by the same membrane in different electrolyte solutions

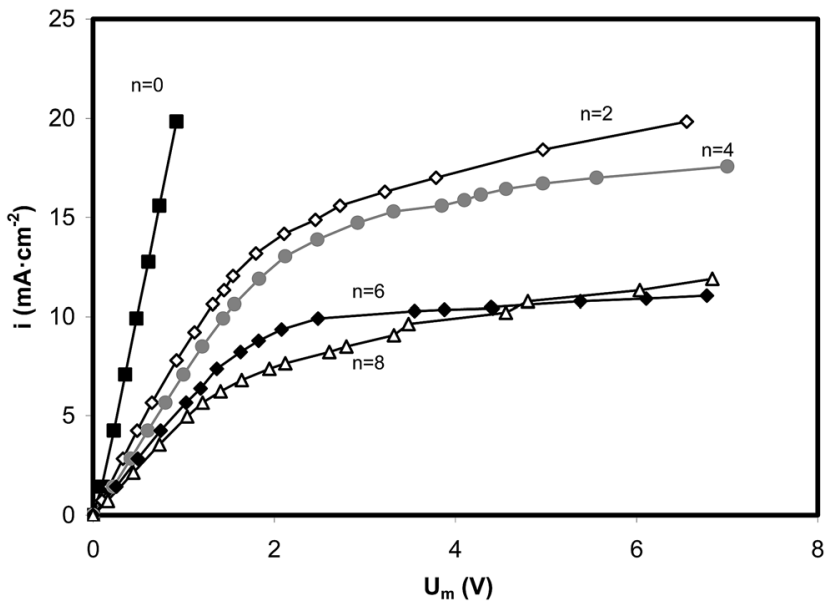

Fig. 11 Current-voltage curves obtained for $0.05 \mathrm{M} \mathrm{HCl}$ solutions.

may indicate that its selectivity for anions under acidic conditions is higher than in neutral media.

Apart from the elucidation of the different regions of membrane behavior, also other relevant parameters can be calculated from the current-voltage curves, which can serve to quantify the properties of the different synthesized membranes. In general it can be seen that the slopes of the quasi-ohmic regions decrease as the number of infiltration cycles increases (see Fig. 10 and 11). The electrical resistance of the different membrane/electrolyte systems, $R_{\mathrm{m}}$, can be calculated from the inverse of the slopes of the quasi-ohmic region; and can be subsequently used to obtain the effective electrical conductivity of the membranes, $k_{\mathrm{m}}$, by using eqn (4):

$$
k_{\mathrm{m}}=\frac{e}{R_{\mathrm{m}}}
$$

In eqn (4), $e$ represents the membrane thickness. In addition, the $i_{\text {lim }}$ values can also be calculated from the intersection of the tangents to the quasi-ohmic region and the plateau. The $k_{\mathrm{m}}$ and $i_{\text {lim }}$ values of each membrane/electrolyte system are presented in Table 1. For a specific electrolyte composition, the $k_{\mathrm{m}}$ values show a general decrease with an increase in the number of infiltration cycles. This trend is strongly correlated with the porosity and pore size distribution of the membranes. As the amount of $\mathrm{ZrO}_{2}$ embedded in the microporous structures increases, the diameter of the membrane pores decreases gradually, especially for the highest ones. Even some pores or the interconnections between some pores may become completely blocked by the infiltration of ion exchanger. Consequently, though more selective, the ionic pathways inside the membrane structure become narrower, fewer and more tortuous. The results obtained indicate that the improvement in the anion selectivity is achieved at the expense of sacrificing the membrane porosity, hence causing a reduction in the membrane conductivity.

As seen in Table 1, the electrolyte composition also has a significant effect on the values of $k_{\mathrm{m}}$, since the electrical conductivity increases with the concentration of $\mathrm{NaCl}$. This 
Table 1 Values of IEC, open porosity and electrochemical parameters as a function of the number of infiltration cycles with hydrated $\mathrm{ZrO}_{2}$

\begin{tabular}{lllllll}
\hline$n$ & & 0 & 2 & 4 & 6 & 8 \\
\hline \multirow{2}{*}{ IEC $\left(\mathrm{meq} \mathrm{g}^{-1}\right)$} & & 0 & 0.030 & 0.051 & 0.070 & 0.070 \\
Open porosity $(\%)$ & 53.6 & 51.8 & 50.5 & 48.5 & 44.8 \\
$0.01 \mathrm{M} \mathrm{NaCl}$ & $k_{\mathrm{m}}\left(\mathrm{mS} \mathrm{cm}^{-1}\right)$ & 0.42 & 0.25 & 0.24 & 0.18 & 0.12 \\
& $i_{\lim \left(\mathrm{mA} \mathrm{cm}^{-2}\right)}-$ & 0.50 & 0.28 & 0.20 & 0.19 \\
$0.05 \mathrm{M} \mathrm{NaCl}$ & $k_{\mathrm{m}}\left(\mathrm{mS} \mathrm{cm}^{-1}\right)$ & 1.95 & 1.34 & 1.26 & 1.13 & 0.88 \\
& $i_{\lim \left(\mathrm{mA} \mathrm{cm}^{-2}\right)}-$ & - & 6.8 & 6.1 & 5.5 \\
$0.1 \mathrm{M} \mathrm{NaCl}$ & $k_{\mathrm{m}}\left(\mathrm{mS} \mathrm{cm}^{-1}\right)$ & 6.65 & 2.40 & 1.91 & 1.25 & 0.91 \\
& $i_{\lim }\left(\mathrm{mA} \mathrm{cm}^{-2}\right)$ & - & - & 9.9 & 7.0 & 6.4 \\
$0.05 \mathrm{M} \mathrm{HCl}$ & $k_{\mathrm{m}}\left(\mathrm{mS} \mathrm{cm}^{-1}\right)$ & 6.56 & 2.58 & 2.18 & 1.61 & 1.50 \\
& $i_{\lim }\left(\mathrm{mA} \mathrm{cm}^{-2}\right)$ & - & 14.9 & 14.6 & 9.9 & 7.4 \\
$0.05 \mathrm{M} \mathrm{NaCl}+$ & $k_{\mathrm{m}}\left(\mathrm{mS} \mathrm{cm}^{-1}\right)$ & 3.79 & 3.03 & 2.79 & 1.86 & - \\
$0.05 \mathrm{M} \mathrm{NaOH}$ & $i_{\lim }\left(\mathrm{mA} \mathrm{cm}^{-2}\right)$ & - & - & - & - & - \\
& & & & & &
\end{tabular}

trend was the expected because an increase in the concentration of ions implies a higher conductivity in the diffusion boundary layers and inside the microporous membrane structure. It is quite remarkable that the conductivities obtained for the $\mathrm{HCl}$ solutions are higher than those obtained for $\mathrm{NaCl}$ solutions of the same concentration. This difference is caused by the higher ionic conductivity of $\mathrm{H}^{+}$ions $\left(3.50 \times 10^{-2} \mathrm{~S} \mathrm{~m}^{2} \mathrm{~mol}^{-1}\right)$ with respect to that of $\mathrm{Na}^{+}$ions $\left(5.01 \times 10^{-3} \mathrm{~S} \mathrm{~m}^{2} \mathrm{~mol}^{-1}\right){ }^{31}$ Despite that both species may be excluded from the membrane phase due to the repulsive forces of the fixed charges, it has to be taken into account that the cations contribute to increase the conductivity of the diffusion boundary layers.

With regard to the $i_{\text {lim }}$ values, this parameter decreases for an increasing number of infiltration cycles. In this case, the $i_{\text {lim }}$ values are clearly correlated with the IEC, since for the uncharged support $(n=0$, IEC $=0)$ the curves did not exhibit a limiting current. However, the membranes having increasing amounts of hydrated $\mathrm{ZrO}_{2}$ possess an enhanced selectivity and the $i_{\lim }$ values decrease gradually with $n$. For a specific membrane system, the $i_{\text {lim }}$ is reached when the concentration gradients at the depleting electrolyte/membrane interface become very high and the vanishing of the concentration of counterions $\left(\mathrm{Cl}^{-}\right)$occurs at the membrane surface. Since membranes with higher selectivity (higher IEC values) have a higher tendency to promote the preferential transport of counterions, the limiting conditions in these membranes are reached at lower values of current density. These results also corroborate the absence of selectivity of the membranes in alkaline media; since the current-voltage curves obtained at basic $\mathrm{pH}$ values were straight and analogous to those obtained for the unmodified supports, and the determination of any $i_{\text {lim }}$ value was not possible. A similar phenomenon was also observed for the membranes with $n=2$ in the cases when the concentration of $\mathrm{NaCl}$ was higher than $0.01 \mathrm{M}$. This fact indicates that the IEC achieved after two infiltration cycles is not sufficient to impart significant anion-selective properties when the electrolyte concentration is higher. The experimental conditions in those cases may not correspond to the Donnan coion exclusion domain, i.e. the concentration of cations in the liquid electrolyte is much higher than the concentration of membrane fixed charges, so that the membrane cannot effectively repel the co-ions. ${ }^{32}$ On the contrary, at more acidic $\mathrm{pHs}$ $(0.05 \mathrm{M} \mathrm{HCl})$ the selectivity of the membranes with $n=2$ is still sufficient to detect the $i_{\text {lim }}$ (see Fig. 11 and Table 1), hence confirming that the selectivity for anions is improved under acidic conditions.

In summary, the present work reports a facile methodology that can be used to produce ion-selective membranes from ceramic materials. This procedure allowed us to obtain membranes with restricted swelling and enhanced chemical durability, which can be implemented as separators in electromembrane reactors applied to treat harsh industrial effluents. The experimental results revealed that the membrane selectivity is mainly determined by the quantity of aggregates of ion exchanger formed inside the microporous ceramic supports. However, the infiltrated particles of hydrated $\mathrm{ZrO}_{2}$ also reduce the porosity of the membranes. This blocking effect seems to imply an increase in the tortuosity and a reduction of the ion-conductive pathways of the electrolyte membranes; thus causing a decrease in their electrical conductivity. In addition, a limit in the number of effective infiltration cycles was found, from which the formation of larger aggregates of hydrated $\mathrm{ZrO}_{2}$ did not entail an increase in the membrane selectivity. In this respect, the balance between membrane selectivity and electrical conductivity can be modulated by optimizing the number of infiltration cycles. In the present study, the optimum number of infiltration cycles was found to be 6 , and further infiltration cycles implied a decrease in the electrical conductivity without achieving a substantial increase in the selectivity for anions. Finally, it must be emphasized that the pore-size distribution resulted determinant for the subsequent deposition of ion exchanger. This issue was facilitated in the present case by the addition of starch as pore former during the synthesis of the microporous ceramic supports.

\section{Conclusions}

The present article reports the synthesis and characterization of novel anion-exchange membranes obtained from the surface functionalization of microporous ceramic supports. The successive infiltration of hydrated $\mathrm{ZrO}_{2}$ particles induced anionselective properties on the microporous supports. The particles of $\mathrm{ZrO}_{2}$ tend to deposit preferentially inside the pores of larger diameter $(>1 \mu \mathrm{m})$, which are formed as a consequence of the addition of starch to the alumina-kaolin matrix. Moreover, the repetition of the infiltration cycle results in the formation of agglomerates of several particles of $\mathrm{ZrO}_{2}$.

When tested in acidic and neutral media; the increase of $U_{\mathrm{m}}$ in the chronopotentiograms, which is associated with the intensification of concentration polarization, reveals a clear correspondence between the load of hydrated $\mathrm{ZrO}_{2}$ and the membrane selectivity for anions. This tendency is confirmed by the detection of limiting current densities and the formation of long plateaus in the current-voltage curves. The electrical conductivity of the different membrane systems decreased with the number of infiltration cycles due to the reduction of the number of ion conducting pathways. When tested with 
mixtures of $\mathrm{NaOH}$ and $\mathrm{NaCl}$; the membrane behavior is similar to that of the uncharged supports, thus indicating that the membranes lack of anion-exchange properties under alkaline conditions. The development of ceramic ion-exchange membranes could make feasible the use of electromembrane processes in industrial applications where the use of polymeric ones is unfeasible due to their weak chemical stability and/or expensive cost.

\section{Acknowledgements}

This work was financially supported by the Ministerio de Economía y Competitividad (Spain) with the project number CTQ2012-3750-C02-01/PPQ and CTQ2012-3750-C02-02/PPQ.

\section{References}

1 T. Xu, J. Membr. Sci., 2005, 263, 1-29.

2 S. Abdu, M. C. Martí-Calatayud, J. E. Wong, M. GarcíaGabaldón and M. Wessling, ACS Appl. Mater. Interfaces, 2014, 6, 1843-1854.

3 J. R. Varcoe, P. Atanassov, D. R. Dekel, A. M. Herring, M. A. Hickner, P. Kohl, A. R. Kucernak, W. E. Mustain, K. Nijmeijer, K. Scott, T. Xu and L. Zhuang, Energy Environ. Sci., 2014, 7, 3135-3191.

4 X. Li, S. Cheng, L. Wang, Q. Long, J. Tao, G. Nie and S. Liao, RSC Adv., 2014, 4, 29682-29693.

5 J. A. Asensio, E. M. Sanchez and P. Gomez-Romero, Chem. Soc. Rev., 2010, 39, 3210-3239.

6 H. Strathmann, Desalination, 2010, 264, 268-288.

7 S. H. Moon and S. H. Yun, Curr. Opin. Chem. Eng., 2014, 4, 25-31.

8 Y. Zheng, X. Gao, X. Wang, Z. Li, Y. Wang and C. Gao, RSC Adv., 2015, 5, 19807-19817.

9 L. Dammak, C. Larchet and D. Grande, Sep. Purif. Technol., 2009, 69, 43-47.

10 S. Koter, P. Piotrowski and J. Kerres, J. Membr. Sci., 1999, 153, 83-90.

11 Y. Iwai, A. Hiroki, M. Tamada, K. Isobe and T. Yamanishi, Radiat. Phys. Chem., 2010, 79, 46-51.

12 X. Li, J. Tao, G. Nie, L. Wang, L. Li and S. Liao, RSC Adv., 2014, 4, 41398-41410.
13 P. Raghavan, X. Zhao, J. K. Kim, J. Manuel, G. S. Chauhan, J. H. Ahn and C. Nah, Electrochim. Acta, 2008, 54, 228-234.

14 M. Raja, N. Angulakshmi, S. Thomas, T. P. Kumar and

A. M. Stephan, J. Membr. Sci., 2014, 471, 103-109.

15 T. Yamaguchi, F. Miyata and S. Nakao, J. Membr. Sci., 2003, 214, 283-292.

16 Y. Dzyazko, Y. Volfkovich, V. Sosenkin, N. Nikolskaya and

Y. Gomza, Nanoscale Res. Lett., 2014, 9, 1-12.

17 A. Clearfield, Chem. Rev., 1988, 88, 125-148.

18 L. A. Rodrigues, L. J. Maschio, R. E. Da Silva and M. L. C. P. Da Silva, J. Hazard. Mater., 2010, 173, 630-636.

19 M. C. Martí-Calatayud, M. García-Gabaldón, V. PérezHerranz, S. Sales and S. Mestre, Desalin. Water Treat., 2013, 51, 597-605.

20 M. García-Gabaldón, V. Pérez-Herranz, E. Sánchez and S. Mestre, J. Membr. Sci., 2006, 280, 536-544.

21 M. C. Martí-Calatayud, M. García-Gabaldón, V. PérezHerranz, S. Sales and S. Mestre, Ceram. Int., 2013, 39, 4045-4054.

22 M. C. Martí-Calatayud, D. C. Buzzi, M. García-Gabaldón, A. M. Bernardes, J. A. S. Tenório and V. Pérez-Herranz, J. Membr. Sci., 2014, 466, 45-57.

23 G. M. Geise, A. J. Curtis, M. C. Hatzell, M. A. Hickner and B. E. Logan, Environ. Sci. Technol. Lett., 2014, 1, 36-39.

24 D. M. Ávila and E. N. S. Muccillo, Thermochim. Acta, 1995, 256, 391-398.

25 Y. Dzyazko, A. S. Rudenko, Y. Yukhin, A. V. Palchik and V. N. Belyakov, Desalination, 2014, 342, 52-60.

26 X. Li, Y. Yu and Y. Meng, ACS Appl. Mater. Interfaces, 2013, 5, 1414-1422.

27 Y. Dzyazko, A. Mahmoud, F. Lapicque and V. N. Belyakov, J. Appl. Electrochem., 2007, 37, 209-217.

28 H. Ohya, K. Masaoka, M. Aihara and Y. Negishi, J. Membr. Sci., 1998, 146, 9-13.

29 W. J. Koros, Y. J. Ma and T. Shimidzu, J. Membr. Sci., 1996, 120, 149-159.

30 M. C. Martí-Calatayud, M. García-Gabaldón and V. PérezHerranz, J. Membr. Sci., 2013, 443, 181-192.

31 D. R. Lide, CRC Handbook of Chemistry and Physics, CRC Press Inc., Boca Raton, 2009.

32 R. Baker, Membrane Technology and Applications, John Wiley \& Sons Ltd., Menlo Park, California, 2004. 